\title{
Toward an Experimental Timing Standards Lab: Benchmarking precision in the real world
}

\author{
RICHARD R. PLANT, NICK HAMMOND, and TOM WHITEHOUSE \\ University of York, York, England
}

\begin{abstract}
Much discussion has taken place over the relative merits of various platforms and operating systems for real-time data collection. Most would agree that, provided great care is taken, many are capable of millisecond timing precision. However, to date, much of this work has focused on the theoretical aspects of raw performance. It is our belief that researchers would be better informed if they could place confidence limits on their own specific paradigms in situ and without modification. To this end, we have developed a millisecond precision test rig that can control and time experiments on a second presentation machine. In this paper, we report on the specialisthardware and software used. We elucidate the importance of the approach in relation to real-world experimentation.
\end{abstract}

There is little doubt that the majority of today's highspeed, high-spec hardware and operating systems are capable of real-time data collection(Finney, 2001; MacInnes $\&$ Taylor, 2001). The caveat is the word capable. As many researchers have pointed out, the dominance of multitasking operating systems makes the picture murky, at best (Myors, 1998, 1999).

To date, focus has concentrated on the raw performance of platforms and operating systems. Many studies have investigated suitability for high-precision timing by using programs that examine the performance of repeating a simple operation within a tight timing loop (e.g., MacInnes \& Taylor, 2001). The effect of stressing the operating system with a background task is a widely accepted variable. Such research, although providing a solid baseline, leaves researchers in the field with the following fundamental question: How does my own paradigm perform in the real world?

Until now, this has been a question that has been extremely difficult to answer. Complete real-world paradigms can often be extremely complex, making use of both visual and auditory stimuli and requiring complex patterns of responses from subjects, with the more being done, the greater the likelihood of lower timing resolution. It is acknowledged that the hardware running the experiment will also be a large factor. What is needed, then, is a rig that can test the majority of paradigms in situ.

To this end, we have developed a generic rig that can be used to control and time a second machine that is being used for presentation-that is, a machine running

The Experimental Timing Standards Laboratory project was funded under Engineering and Physical Sciences Research Council Grant GR/N38350/01. The project Web site can be visited at http://www. psychology.ltsn.ac.uk/ETSL/. Correspondence concerning this article should be addressed to R. R. Plant, Department of Psychology, University of York, York YO10 5DD, England (e-mail: r.plant@psych.york.ac.uk). the experimental paradigm under test. The results of testing can be used to inform a researcher how well their hardware, experimental software, and paradigm have performed as a cohesive unit. This work has been conducted under a funded project called the Experimental Timing Standards Laboratory (ETSL; see http://www.psychology. ltsn.ac.uk/ETSL/).

In this paper, we report on the specialist hardware and software we have developed, the rationale behind it, and studies validating the performance of the test rig. A forthcoming paper will describe the set of benchmarks we are using to assess both commercial and noncommercial experiment generators.

\section{THE HARDWARE}

Our ETSL benchmarking rig is made up of various components. These consist of an instrumented PC responsible for control and timing (CAT) and a custom-built breakout box. The breakout box offers various interfaces to sensors and standard input devices that interact with a second computer (PRES, for presentation) running a paradigm we are interested in (see Figures 1, 1A, and 1B).

The key issue when building a test rig is to ensure that it actually does what it is meant to do-that is, operate consistently with millisecond or better precision without interfering in any way with the hardware and software under test. The only way to hope to achieve this is to make use of specialist data acquisition hardware and timing boards. This means that data are acquired through a high-rate sampling board, with timings recorded independently of the host PC and operating system.

To establish a solid real-time platform, we chose to use National Instruments (http://www.ni.com) data acquisition (DAQ) and timing boards within our CAT PC. For high-speed DAQ, we made use of the NI 6533. The 6533 performs single-point I/O, pattern I/O, and high-speed 

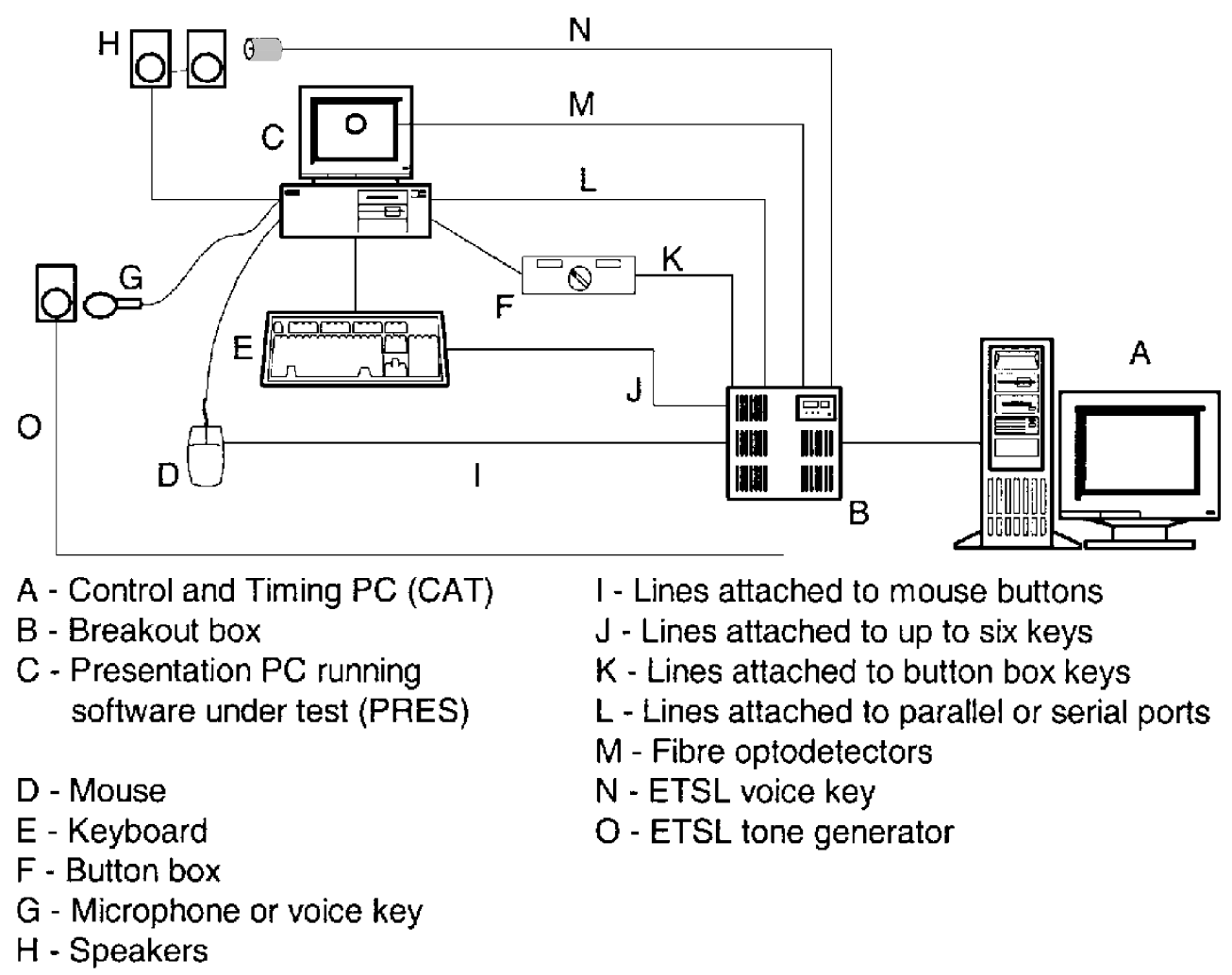

I - Lines attached to mouse buttons

$J$ - Lines attached to up to six keys

$K$ - Lines attached to button box keys

$L$ - Lines attached to parallel or serial ports

$M$ - Fibre optodetectors

$N$ - ETSL voice key

$\mathrm{O}$ - ETSL tone generator

Figure 1. An overview of CAT and PRES.

data transfer, using a wide range of handshaking protocols at speeds of up to $76 \mathrm{MB} / \mathrm{sec}$ (PCI). It has a pattern detection resolution of $60 \mathrm{nsec}$ and change detection resolution of $150 \mathrm{nsec}$. Start and stop TTL triggers can be on rising or falling edge or digital pattern, with a minimum width for edge triggers of $10 \mathrm{nsec}$.

For timing, a NI 6601 was used. The PCI-6601 offers four 32-bit counter/timers and up to 32 lines of TTL/ CMOS-compatible digital I/O. A wide variety of counter/ timer tasks can be performed, including encoder position measurement, event counting, period measurement, pulse-width measurement, pulse generation, pulse-train generation, and frequency measurement. A clock frequency of $100 \mathrm{kHz}$ was used throughout, giving a potential rollover time of $11.93 \mathrm{~h}$. The 6601 has a base clock accuracy of $50 \mathrm{ppm}( \pm 0.005 \%)$ over temperature. The minimum pulse and gate duration are $5 \mathrm{nsec}$, or 50 nsec when using the Real Time Send Interface (RTSI).

Within CAT, both boards were connected, using a RTSI ribbon cable, allowing the 6533 to trigger a timestamp on the 6601 counter/timer. The 6601 has the ability to record data straight to an applications memory, using DMA transfers. CAT itself was a 900-MHz Athlon with $512 \mathrm{MB}$ of RAM, an $80 \mathrm{~GB}$ hard drive, and an AGP Leadtek Geforce $2 \mathrm{Mx}$ with $32 \mathrm{MB}$. The only PCI slots filled were with the two NI boards described above. No network or sound card was present (on-board sound was disabled). Windows 2000 Professional SP1 provided the operating system. NI DAQ drivers, Version 6.8, were used throughout to access the NI cards.

PRES was another $900-\mathrm{MHz}$ Athlon with $128 \mathrm{MB}$ of RAM, a 20-GB hard drive, and an ATI Rage Pro (AGP) with $16 \mathrm{MB}$ of video RAM. Sound was catered for by an on-board AC97 compatible chipset. Windows 98R2 provided the operating system with MS DirectX8.0a installed. Again, no network card was present.

In order to enable CAT to control and time experimental paradigms running on PRES, various sensors and output devices were connected to a custom-built breakout box, which was, in turn, connected by an SCSI cable to the 6533 board of CAT (see Figure 2). Standard input devices, such as keyboards, mice, and response boxes, could be patched to port $\mathrm{C} 0 \sim 7$ via $2.5-\mathrm{mm}$ jack plugs. In the case of the keyboard, a permanently wired ribbon cable allows up to six keys to be controlled. All eight lines have an activity LED. The input devices themselves were connected to PRES as normal. Figure 3 shows a schematic of the breakout box interface.

Up to eight optodetectors (1-mm fiber cable) can be attached to the screen of PRES via suction cups in order to detect visual stimuli (port A0 7). A sensitivity potentiometer and activity LED are provided for each line. Electronics within the box provide a TTL signal for the 6553 board. 

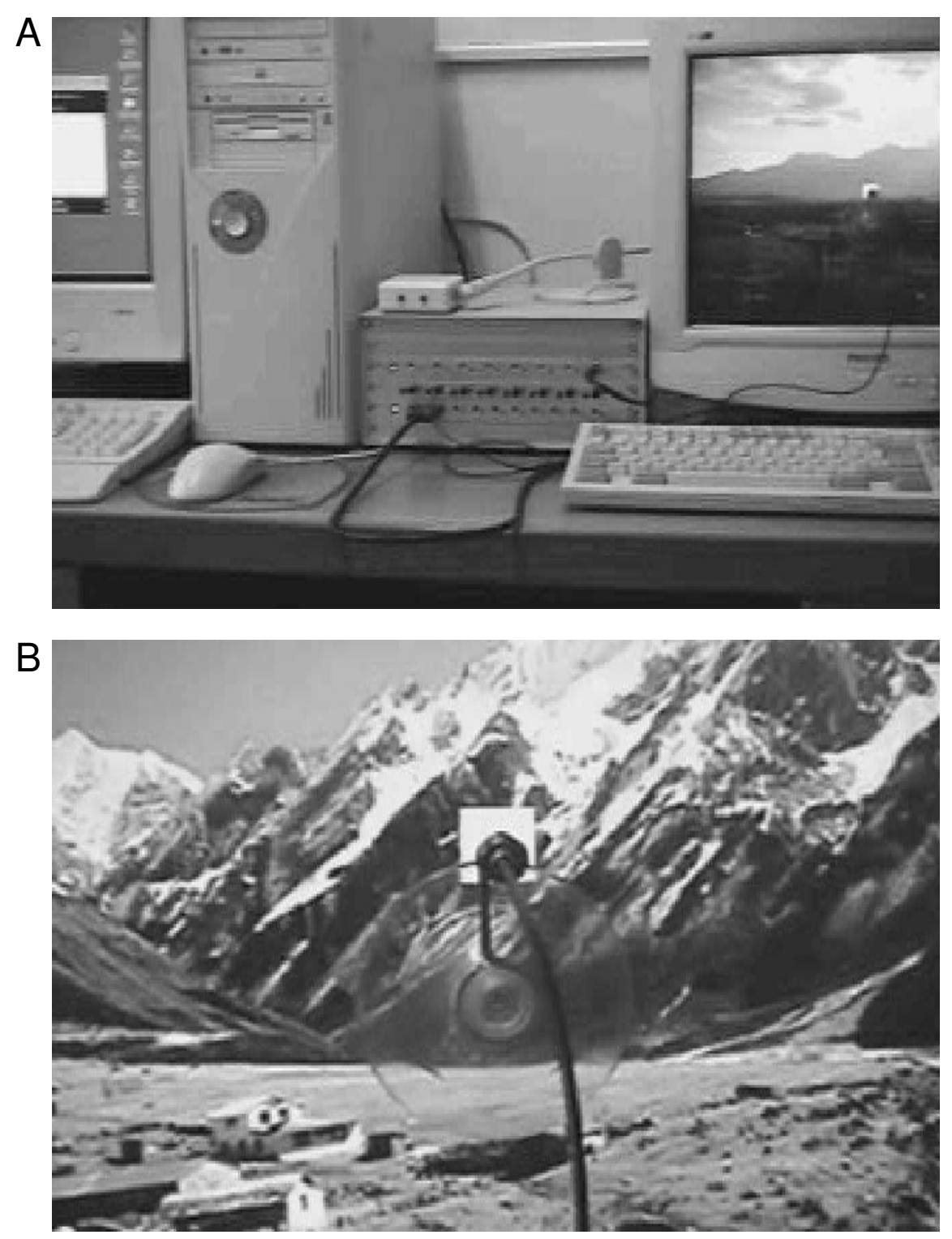

Figure 1. (A) Here CAT can be seen on the left, with the breakout box center and PRES on the right. In this test, PRES is instructed to display a visual stimulus for a set duration and wait for a response via a microphone. When CAT "sees" the leading edge (first refresh) of the visual stimulus, it waits a set interval, then generates a 100-msec tone, using the ETSL tone generator (top left of the breakout box). (B) Fiber optodetectors are attached to the screen of PRES, using suction cups.

A multifunction port out (D0 3) provides four digital lines via $3.5-\mathrm{mm}$ jack plugs. Usually, these lines control our custom-built tone generators used for testing the audio response capabilities (voice key) of software being tested on PRES.

Finally, a multifunction port in (B0 3) typically enables input from our custom-built voice key in response to audio events generated by software running on PRES. Electronics within the voice key convert the analogue signal to a TTL one, suitable for input into the breakout box and ultimately to CAT. Activity LEDs are provided for ports B0 3 and D0 3.

\section{THE CONTROL AND TIMING SOFTWARE}

Having designed and built a set of custom hardware devices capable of triggering events and capturing data from a variety of external sources, the next stage was to write a set of software tools to manage the process.

As was stated above, our goal was to create a generic rig that could be used to test most experimental paradigms in situ, running on any platform. This entailed writing CAT software that could simulate event sequences, such as keypresses, capture events, such as visual stimulus displays, and simulate events after detecting a stim- 


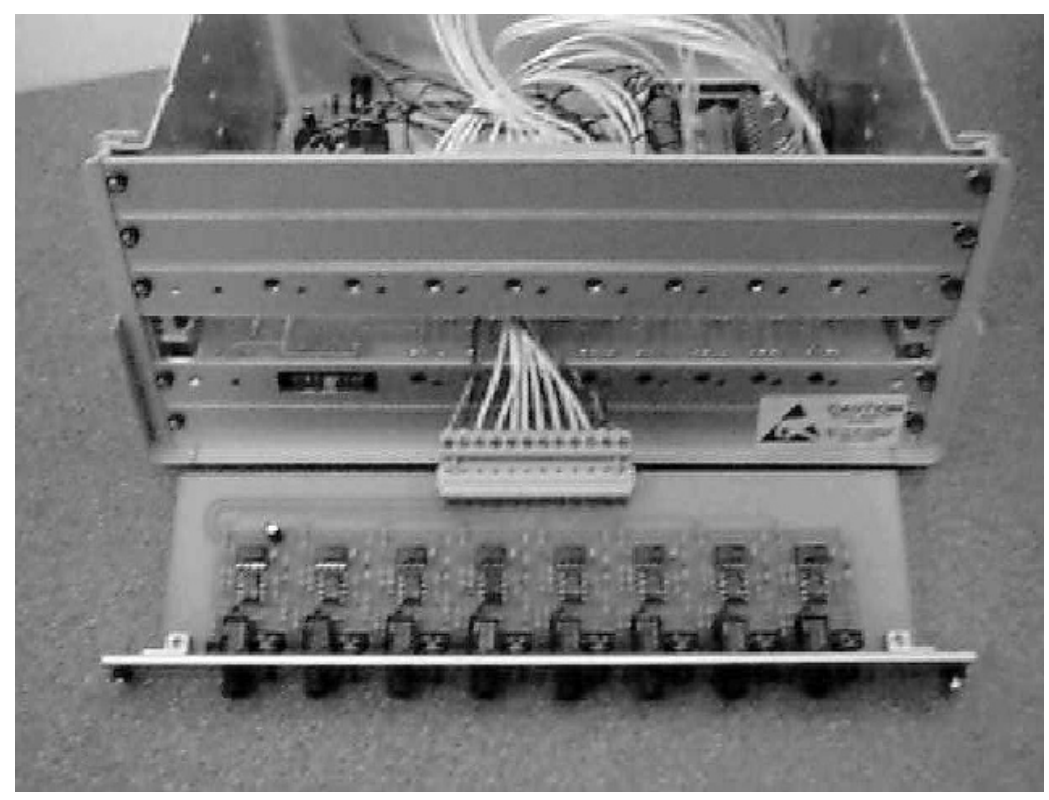

Figure 2. The breakout box with the optodetector card removed.

ulus, such as a keypress after detecting the presence of a visual stimulus.

All software uses Microsoft Visual Basic Professional, Version 6.0 SP3, combined with National Instruments (http://www.ni.com) component works and an NI DAQ driver, Version 6.8, to provide access to the NI boards.

\section{Event Generation Software}

Our Event Generation (EG) was used on CAT to generate responses according to a preprogrammed schedule. The purpose of EG was to simulate a set of responses that could be fed into PRES in order to simulate a human making a sequence of responses. This makes it possible to compare the sequence of responses recorded by the software under test with one that has been accurately produced. Thus, response registration accuracy can be evaluated. A typical psychological paradigm might be a simple finger-tapping experiment.

EG allows responses to be generated across all eight lines of a port on the 6533 DIO card. This enables up to eight simultaneous keypresses to be fed into PRES. Typically, only one line was used on the keyboard, mouse, or tone generator ports.

In addition to being able to operate up to eight lines, individual event onset times and duration can be controlled. This enables complex patterns of response actions to be constructed. Generally, these more complex sequences were created in Excel and loaded into the

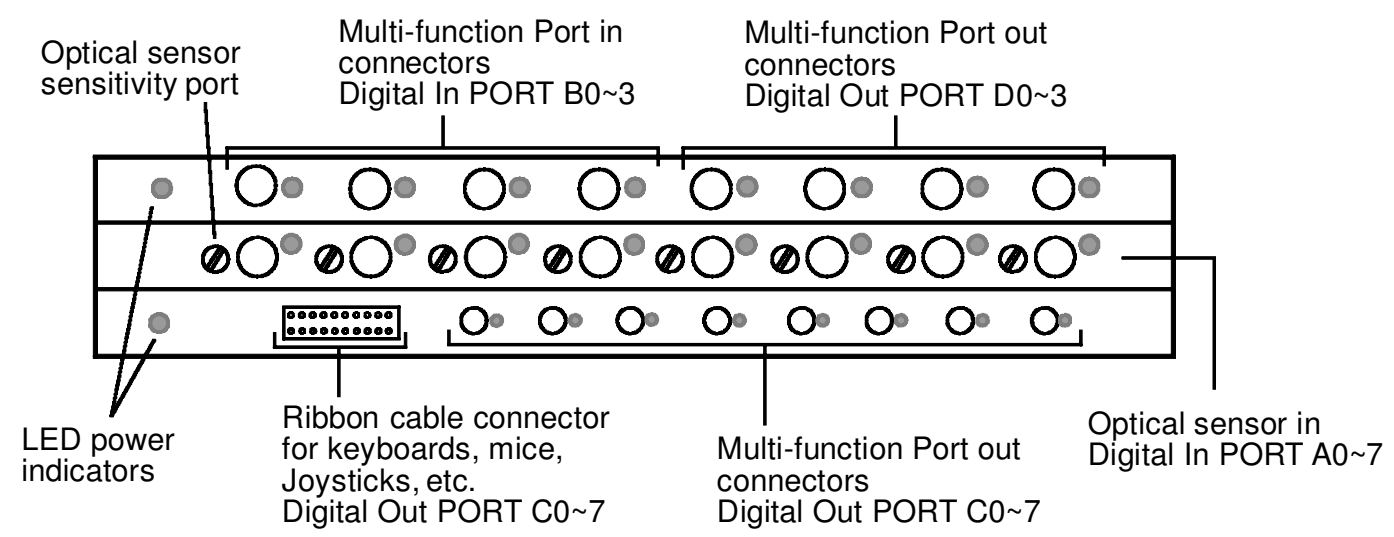

Figure 3. A schematic of the breakout box interfaces to the 6533 board. 


\begin{tabular}{|c|c|c|c|c|}
\hline Event & Block & Dnset [ms] & Port Val & Duration [ms] \\
\hline 1 & 1 & 1000 & 191 & 100 \\
\hline 2 & 1 & 1200 & 191 & 100 \\
\hline 3 & 1 & 1400 & 191 & 100 \\
\hline 4 & 1 & 1600 & 191 & 100 \\
\hline 5 & 1 & 1800 & 191 & 100 \\
\hline 6 & 1 & 2000 & 191 & 100 \\
\hline 7 & 1 & 2200 & 191 & 100 \\
\hline 8 & 1 & 2400 & 191 & 100 \\
\hline 9 & 1 & 2600 & 191 & 100 \\
\hline 10 & 1 & 2800 & 191 & 100 \\
\hline
\end{tabular}

Figure 4. A simple Event Generation Schedule.

schedule before being run. All event timing is controlled by the 6601 counter/timer. To ensure accuracy, EG is run in real-time priority mode.

In Figure 4, 10 events are to be generated. Here, events are generated from 1,000 msec onwards. Each has its onset time specified, along with a port value (191 actually means press the "B" key) and a duration. This schedule will simulate pressing the " $\mathrm{B}$ " key on PRES's keyboard 10 times, with a 100-msec key-down duration and a 100-msec key-up duration.

\section{Visual Stimulus Capture and Digital Stimulus Capture}

Visual Stimulus Capture (VSC) and Digital Stimulus Capture (DSC) are essentially identical pieces of software, with the same timing characteristics, with the exception that the latter allows you to capture events across any digital port, rather than just across the optodetectors. VSC is used on CAT to measure the visual stimulus presentation accuracy of any piece of psychological software being run on PRES (DSC could capture audio events by using our voice key, for example). This is done in terms of the duration of each visual stimulus and the interstimulus gap (ISG) between them. In short, it constantly records an incoming data stream on the optodetectorport of the 6533. Up to eight optodetectors can be used simultaneously.

Up to 8.5 min worth of data can be collected as a constant data stream. This takes up around $130 \mathrm{MB}$ of RAM, available on CAT. No data is spooled to the hard drive until the capture time limit specified has been reached. VSC is run in real-time priority mode to ensure accuracy.

VSC has been independently validated as being able to collect over six samples per millisecond across eight optical sensors, with a known accuracy when using polling. As each sample is collected, it is time-stamped by reading from the 6601 counter/timer card. Internally, only a hex port value and time stamp are recorded per sample, to increase speed and lower memory requirements.
Once the capture time limit is reached, a Comma Separated Value (CSV) file is dumped at the location specified. Each line in the file represents one sample across a port of the 6533. Owing to the high sampling rates, this means that files totaling many hundreds of megabytes can be generated. Files generated are typically larger than their memory footprint, since when spooling to disk occurs, some preprocessing of the data is done.

Since the sampling rate is so high, one can see individual pixels being illuminated where the beam scans down the monitor. Obviously such a volume of data cannot be analyzed by hand. To this end a Captured Data Parser (CDP) was written.

\section{Visual Stimulus Capture and Response and Digital Stimulus Capture and Response}

Visual Stimulus Capture and Response (VSCAR) is used on CAT to measure visual presentation accuracy of any psychological software being run on PRES. This function of VSCAR works in exactly the same way as that described for VSC. The key difference is that a simulated response sequence can be programmed. Events can be simulated by CAT at known intervals after the first refresh (leading edge) of a visual stimulus is detected on PRES. In essence, this means that we can measure both presentation and response accuracy. Responses can be generated to any line of any port of the 6533 and can have any duration. For example, a visual stimulus on PRES could trigger a key-down event to be generated for $50 \mathrm{msec}$.

Digital Stimulus Capture and Response (DSCAR) is an identical application bar that can capture from any port of the 6533. For example, it would be used if you wished to capture a stream of audio events generated by some psychological software and then to simulate keypresses at known intervals after or during those events.

In a similar way to VSC, a capture file can be specified along with the capture time in milliseconds. Again, 


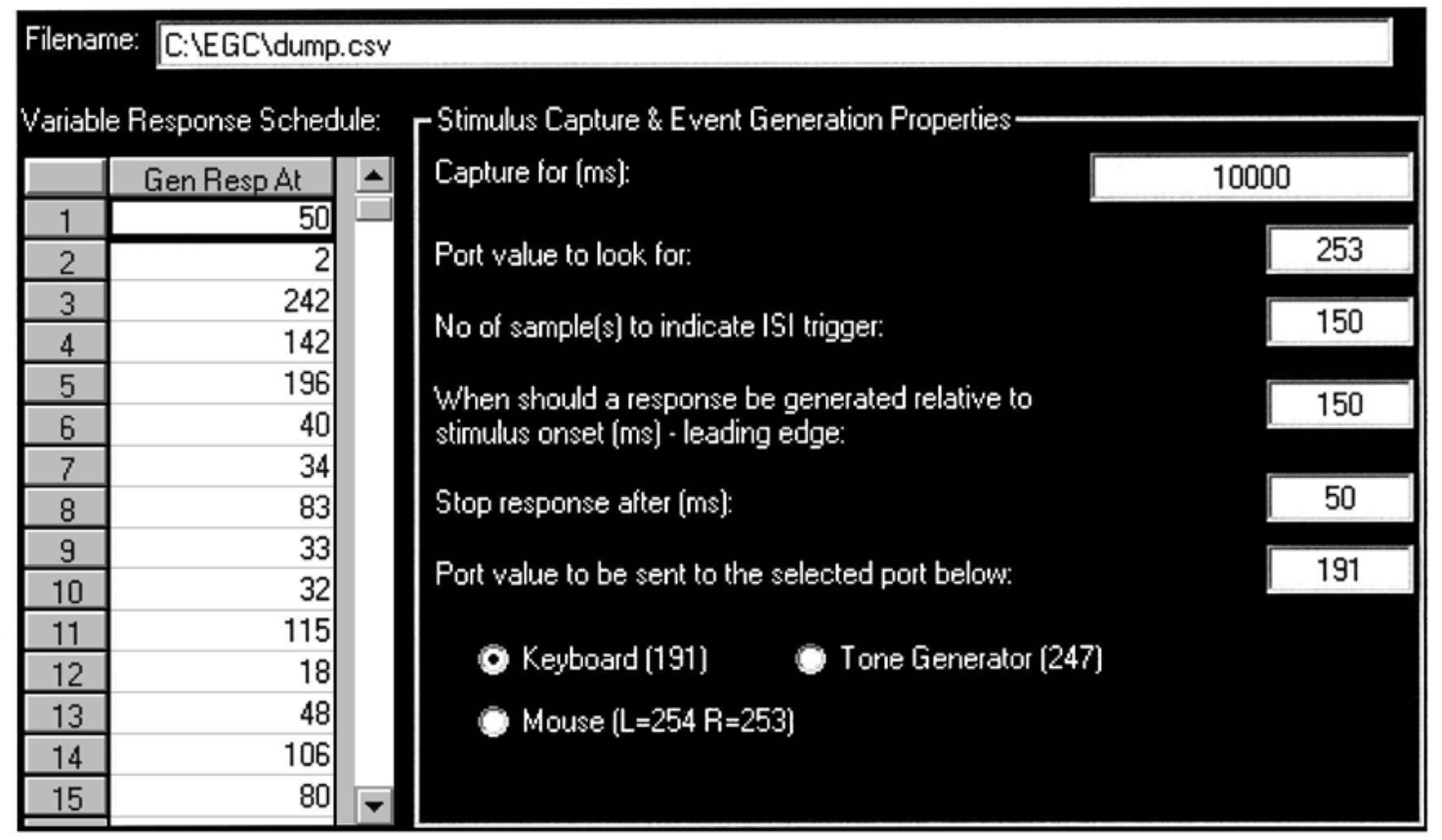

Figure 5. A sample stimulus-response schedule.

to ensure accuracy, VSCAR is run in real-time priority mode.

Other options allow specification of the port value to be "looked" for. An example is shown in Figure 5, where an event on port 253 will trigger a response according to the variable response schedule shown in the spreadsheet to the left. A port value of 253 means that Line 1 of the optodetectors have "seen" a visual stimuli on PRES. Since each refresh is seen as a distinct event block, the number of samples that indicate an ISG is also entered. This figure is the number of 0 (zero) samples (six per millisecond, with our current hardware using polling) that must occur to indicate that a visual stimulus has actually gone off and is not just in the process of being refreshed, ready to be displayed on the next monitor scan. For our purposes, this cannot be less than two refreshes at $100 \mathrm{~Hz}$, or $20 \mathrm{msec}$, or the number of samples that is greater than that obtainable in one refresh.

The "stop response after" figure specifies for how long a response (such as a keypress) will be generated. For example, for the first visual stimulus "seen" on PRES, a response will be generated $50 \mathrm{msec}$ after the leading edge sample; this will be a keyboard event that lasts for $50 \mathrm{msec}$. Once a suitable ISG has passed, a second visual stimuli is displayed. A response will be generated $2 \mathrm{msec}$ after the leading edge and will last for $50 \mathrm{msec}$, and so on. Typically, single keyboard events, mouse-down events, and tones are generated in order to simulate responses to visual stimuli displayed on PRES. However, DSCAR can generate a response relative to any digital stimulus detected, such as an auditory stimulus.
In order to confirm that the psychological software running on PRES is performing to specification, three files are examined. The first is the file holding the responses captured by the psychological software running on PRES. This always includes response times and may include details of stimuli presentation. To be $100 \%$ accurate, response times should match those of the response schedule. However this will not ever be precisely the case, since each input device has its own associated response lag. This is additive when combined with any error introduced by the psychological software itself, plus any effect the operating system may have.

The second file that is captured by VSCAR or DSCAR is the CSV file in which each visual stimulus appearance and duration is captured. The captured values can be compared with expected values.

The final file is the response schedule itself. In effect, these figures represent the response times that ideally should have been detected. All three files are loaded into a customized Excel spreadsheet for analysis. From here, observed minus expected values can be computed and graphs plotted that illustrate how good a given piece of software is at displaying stimuli and detecting responses.

\section{Collected Data Parser}

The ETSL bench software generates more raw data than can be handled by spreadsheet software, and so we needed to write our own parsing software. This has the task of removing samples where nothing happens-for example, where an image is not being displayed on the screen of PRES. This dramatically reduces the size of the 


\begin{tabular}{|c|c|c|c|c|c|c|c|c|c|c|c|c|c|c|}
\hline & A & B & C & D & $\mathbf{E}$ & F & G & $\mathbf{H}$ & I & $\mathbf{J}$ & K & $\mathbf{L}$ & M & $\mathbf{N}$ \\
\hline 13486 & Event ID & Dec Val & LO & $\mathrm{L} 1$ & $\mathrm{~L} 2$ & $\mathrm{~L} 2$ & $\overline{\mathrm{L} 4}$ & L5 & L6 & L7 & Bin & Elapsed CT & Elapsed ms & Response ms \\
\hline 13487 & 13486 & 255 & 0 & 0 & 0 & 0 & 0 & 0 & 0 & 0 & 0 & 2.43637 & 2436.37 & 0 \\
\hline 13488 & 13487 & 255 & 0 & 0 & 0 & 0 & 0 & 0 & 0 & 0 & 0 & 2.43655 & 2436.55 & 0 \\
\hline 13489 & 13488 & 255 & 0 & 0 & 0 & 0 & 0 & 0 & 0 & 0 & 0 & 2.43672 & 2436.72 & 0 \\
\hline 13490 & 13489 & 255 & 0 & 0 & 0 & 0 & 0 & 0 & 0 & 0 & 0 & 2.43691 & 2436.91 & 0 \\
\hline 13491 & 13490 & 255 & 0 & 0 & 0 & 0 & 0 & 0 & 0 & 0 & 0 & 2.43709 & 2437.09 & 0 \\
\hline 13492 & 13491 & 255 & 0 & 0 & 0 & 0 & 0 & 0 & 0 & 0 & 0 & 2.43726 & 2437.26 & 0 \\
\hline 13493 & 13492 & 255 & 0 & 0 & 0 & 0 & 0 & 0 & 0 & 0 & 0 & 2.43744 & 2437.44 & 0 \\
\hline 13494 & 13493 & 255 & $\overline{0}$ & 0 & $\overline{0}$ & 0 & 0 & 0 & 0 & $\overline{0}$ & 0 & 2.43761 & 2437.61 & 2437.61 \\
\hline 13495 & 13494 & 255 & 0 & 0 & 0 & 0 & 0 & 0 & 0 & $\overline{0}$ & 0 & 2.43858 & 2438.58 & 0 \\
\hline 13496 & 13495 & 255 & 0 & 0 & 0 & 0 & 0 & 0 & 0 & 0 & 0 & 2.43881 & 2438.81 & 0 \\
\hline 13497 & 13496 & 255 & 0 & 0 & 0 & 0 & 0 & 0 & 0 & 0 & 0 & 2.439 & 2439 & 0 \\
\hline 13498 & 13497 & 255 & 0 & 0 & 0 & 0 & 0 & 0 & 0 & 0 & 0 & 2.43918 & 2439.18 & 0 \\
\hline 13499 & 13498 & 255 & 0 & 0 & 0 & 0 & 0 & 0 & 0 & 0 & 0 & 2.43935 & 2439.35 & 0 \\
\hline 13500 & 13499 & 255 & 0 & 0 & 0 & 0 & 0 & 0 & 0 & 0 & 0 & 2.43953 & 2439.53 & 0 \\
\hline 13501 & 13500 & 255 & 0 & 0 & 0 & 0 & 0 & 0 & 0 & 0 & 0 & 2.43971 & 2439.71 & 0 \\
\hline 13502 & 13501 & 255 & 0 & 0 & 0 & 0 & 0 & 0 & 0 & 0 & 0 & 2.4399 & 2439.9 & 0 \\
\hline 13503 & 13502 & 255 & 0 & 0 & 0 & 0 & 0 & 0 & 0 & 0 & 0 & 2.44008 & 2440.08 & 0 \\
\hline 13504 & 13503 & 255 & 0 & 0 & 0 & 0 & 0 & 0 & 0 & 0 & 0 & 2.44025 & 2440.25 & 0 \\
\hline 13505 & 13504 & 255 & 0 & 0 & 0 & 0 & 0 & 0 & 0 & 0 & 0 & 2.44043 & 2440.43 & 0 \\
\hline 13506 & 13505 & 253 & 0 & 1 & 0 & 0 & 0 & 0 & 0 & 0 & 1000000 & 2.4406 & 2440.6 & 0 \\
\hline 13507 & 13506 & 253 & 0 & 1 & 0 & 0 & 0 & 0 & 0 & 0 & 1000000 & 2.44078 & 2440.78 & 0 \\
\hline 13508 & 13507 & 253 & 0 & 1 & 0 & 0 & 0 & 0 & 0 & 0 & 1000000 & 2.44096 & 2440.96 & 0 \\
\hline 13509 & 13508 & 253 & 0 & 1 & 0 & 0 & 0 & 0 & 0 & 0 & 1000000 & 2.44114 & 2441.14 & 0 \\
\hline 13510 & 13509 & 253 & 0 & 1 & 0 & 0 & 0 & 0 & 0 & 0 & 1000000 & 2.44131 & 2441.31 & 0 \\
\hline 13511 & 13510 & 253 & 0 & 1 & 0 & 0 & 0 & 0 & 0 & 0 & 1000000 & 2.44149 & 2441.49 & 0 \\
\hline 13512 & 13511 & 255 & 0 & 0 & 0 & 0 & 0 & 0 & 0 & 0 & 0 & 2.44166 & 2441.66 & 0 \\
\hline 13513 & 13512 & 255 & 0 & 0 & 0 & 0 & 0 & 0 & 0 & 0 & 0 & 2.44184 & 2441.84 & 0 \\
\hline 13514 & 13513 & 255 & 0 & 0 & 0 & 0 & 0 & 0 & 0 & 0 & 0 & 2.44202 & 2442.02 & 0 \\
\hline
\end{tabular}

Figure 6. Example captured data showing one sample per row.

dataset, since each blank refresh at $100 \mathrm{~Hz}$ represents 60 samples. The parser also has the job of blocking refresh groups together in the case of visual stimuli. Put simply, if an image is displayed for $100 \mathrm{msec}$, this will actually consist of 10 groups of 6 samples detected by the optodetector. The parser groups these together to give leading and trailing edges and, hence, duration. An example CDP screen is shown in Figure 6. Here, the group of six "100000" entries under the "Bin" column show where pixels under a fiber optodetector were illuminated (the phosphor persistence makes them stay on for around $1 \mathrm{msec})$.

This actual example is from a VSCAR session. Here, the first response is scheduled to be fed into the keyboard of PRES 1,567 msec after the leading edge (first positive sample in the initial refresh) of the visual stimuli first appears on the monitor. Earlier in the sample sequence (not shown), we can see the leading edge of the first stimulus refresh is at $870.62 \mathrm{msec}$. Once VSCAR has detected this, it attempts to wait until exactly $1,567 \mathrm{msec}$ have elapsed. Later in the capture file, we can see that a response was generated at $2,437.61 \mathrm{msec}$ after the start of the run.

So, to check that the response was generated at the correct time, we simply subtract 870.62 from $2,437.61$, to give us $1,566.99 \mathrm{msec}$. This internal validity check helps ensure that a response was simulated by CAT at the correct interval, as specified by the response schedule. Here, we are early by $0.01 \mathrm{msec}$ from the 1,567-msec target, which can be considered insignificant (this is always achieved with submillisecond precision). Responses within a schedule are always relative to the leading edge of a given stimulus, whether it be visual or digital. By comparing the condensed data files culled by CDP with known display intervals programmed into PRES, we can examine whether the stimuli were displayed at the correct times and for the correct durations. In addition, we can compare the time at which we generated simulated responses with that recorded by the psychological paradigm running on PRES. Any differences over $1 \mathrm{msec}$ will be error introduced by the software running on PRES.

\section{VALIDATION}

In order to ensure that our rig would function reliably with millisecond precision, two pieces of standalone bench equipment were used: a Hewlett Packard (Agilent) 33120A Function/Arbitrary Waveform Generator (http:// we.home.agilent.com/) and a Tektronix Digital Phosphor Oscilloscope (DPO) Model TDS 3034 (http://www. tektronix.com/). 


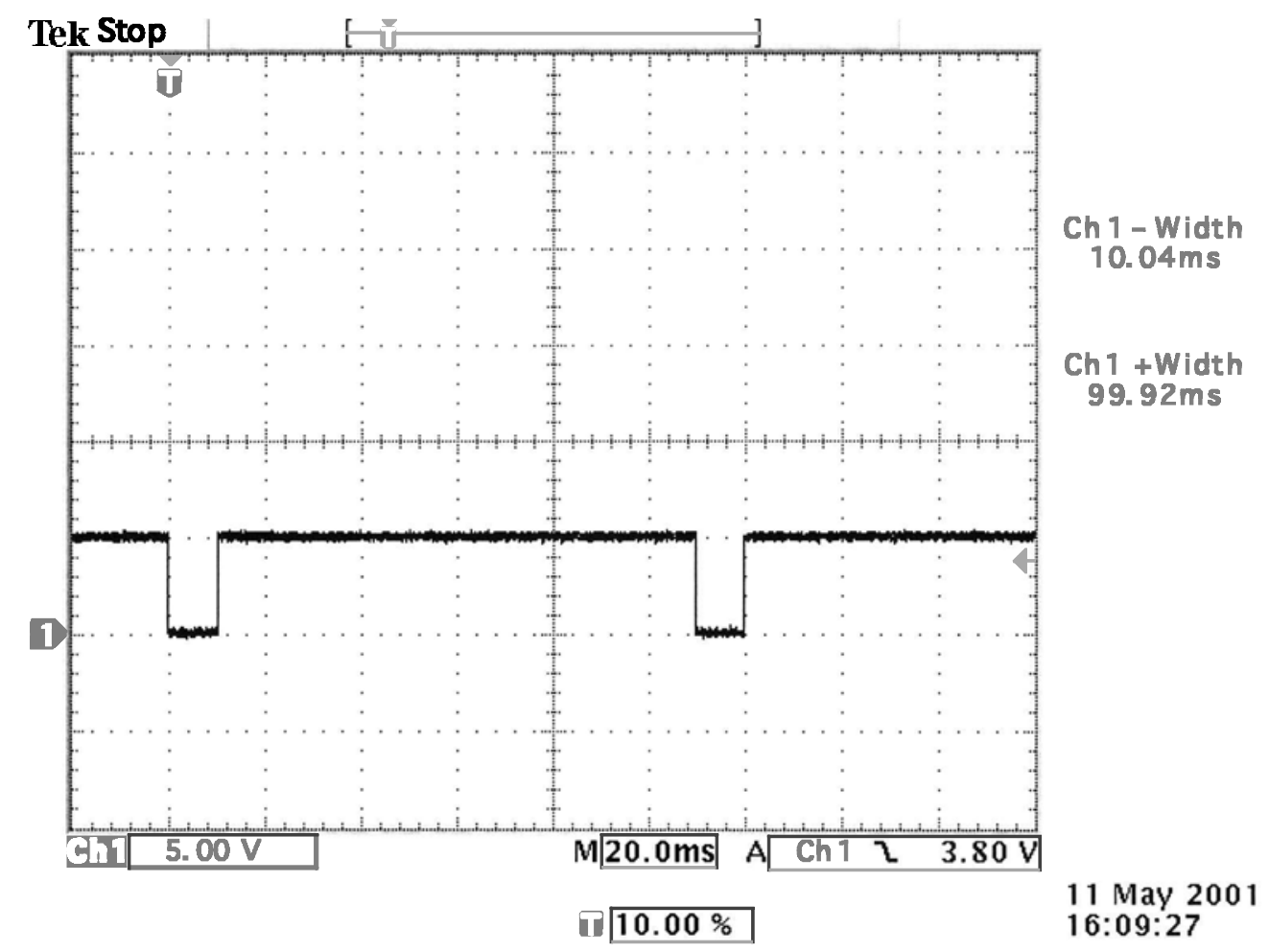

Figure 7. A screen dump from the Tektronix Digital Phosphor Oscilloscope, showing a worst case measure.

The function generator was used to generate accurate digital pulses that could be used to test either custombuilt hardware or benchmarking software. The DPO was used to record events that were either generated by our software or triggered by the 33120A. With both pieces of equipment operating at the nanosecond level, all measures are considered to be extremely accurate. The DPO has a built-in floppy disk to which screen images can be saved, which proved ideal for recording tolerances. A simple worked example shows how our EG software was validated in this case (see Figure 7). EG was configured to simulate a continuous stream of 10 -msec events separated by a $100-\mathrm{msec}$ off periods. These could be thought of as very short key-down and key-up periods on a response box plugged into PRES and patched to CAT. Using this schedule, the following values were obtained over a 2-min period: best case, 10 -msec event and $100 \mathrm{msec}$ off, and worst case, 10.04-msec event and $99.92 \mathrm{msec}$ off, giving a jitter of -0.08 to $+0.04 \mathrm{msec}$ for our EG software. EG was running in real-time priority mode (no disk, keyboard, or mouse access allowed by the operating system) on CAT throughout.

As can be seen, EG when running in real-time priority is capable of submillisecond accuracy, even with worst case values.

DSC and VSC were tested by using the 33120A to generate a stream of pulses $100 \mathrm{msec}$ wide, followed by a $100-\mathrm{msec}$ interval. DSC was used to capture these events over a 2-min period. Jitter was calculated by analyzing the captured data in comparison with expected values.

DSCAR and VSCAR were tested by using both the 33120A and the DPO. The 33120A was set up to pro-

Table 1

Accuracy and Consistency of the ETSL Benchmarking Rig

\begin{tabular}{lc}
\hline \multicolumn{1}{c}{ Software } & Jitter (msec) \\
\hline Event Generation & -0.08 to +0.04 \\
Digital Stimulus Capture (stimulus durations) & -0.36 to -0.02 \\
Digital Stimulus Capture (stimulus gaps) & -0.05 to +0.30 \\
Visual Stimulus Capture & -0.05 to +0.30 \\
Digital Stimulus Capture and Response (stimulus durations) & -0.30 to +0.05 \\
Digital Stimulus Capture and Response (stimulus gaps) & -0.04 to +0.31 \\
Digital Stimulus Capture and Response (simulated response accuracy from target) & -0.84 to -0.58 \\
Visual Stimulus and Response & -0.84 to -0.58 \\
\hline
\end{tabular}

Note-All jitter values were calculated from worst case measures. 
Table 2

Hardware Timing Characteristics of the ETSL Breakout Box

\begin{tabular}{ll}
\hline \multicolumn{1}{c}{ Interface } & Switch Delay \\
\hline $\begin{array}{l}\text { Eight-channel input/output card, } \\
\text { arranged as four input and four output lines } \\
\text { (for input from our custom built voice key and } \\
\text { output for custom-built tone generators) }\end{array}$ & $<1 \mathrm{nsec}$ \\
$\begin{array}{l}\text { Eight-channel optical input card } \\
\begin{array}{l}\text { Eight-channel opto-isolated output card } \\
\text { turn on (for simulating switch operations in }\end{array}\end{array}$ & $\mathrm{TBD}$ \\
keyboards, mice, joysticks, etc.) & $<150 \mu \mathrm{sec}$ \\
\hline $\begin{array}{l}\text { Note-Both the eight-channel I/O card and the eight-channel opto-isolated } \\
\text { output card can be used with any TTL based device. }\end{array}$ &
\end{tabular}

duce a stream of pulses $50 \mathrm{msec}$ wide, followed by a 100 -msec interval. The $33120 \mathrm{~A}$ was connected to the DPO so that we could track when these pulses occurred. A lead connected a breakout box digital line to the DPO so that events generated under our control could be seen in relation to the pulse. DSCAR was programmed to simulate a response $25 \mathrm{msec}$ after the leading edge of the $50-\mathrm{msec}$ pulse for a duration of $50 \mathrm{msec}$. Again, we compared expected with observed values over a 2 -min period.

We were advised by our electronics expert (third author) that timing characteristics would remain stable over longer periods and across varying timing schedules, owing to the NI boards used. This meant that we were able to be confident of the validity of our measures without testing a very large number of permutations.

A summary of the timing characteristics of each piece of ETSL software used to benchmark software running on PRES is shown in Table 1. These measures were taken by using the HP 33120A/DPO through the breakout box, so in effect, they are a summation of the contribution made to timing from each component of the rig.

As part of our ongoing validation study, the individual interfaces offered to and from the breakout box itself were benchmarked - that is, the electronics that converted analogue signals to digital pulses. Table 2 summarizes their timing characteristics.

Given that no worst case timing measures were above $1 \mathrm{msec}$ when standard bench equipment was used, we can be confident in timings obtained when testing paradigms running on PRES.

It is important to note that validation timings relate to the rig as a whole, and not to individual components, such as hardware. So validation timings are based on the sum contribution of ETSL measurement software delay + NI board delay + breakout box delay + sensor/output device delay + any OS factors + any other unknown factors. Note that output device refers only to custom-built hardware, and not to standard keyboards, mice, and so on.

\section{CONCLUSION}

Rather than relying on raw performance benchmarking of hardware and operating system, we have demonstrated that it is possible to build a test rig that can evaluate the timing properties of most paradigms in situ, without modification.

However, this requires substantial effort in that complex custom hardware has to be designed and built, control and analysis software written, and the whole rig independently validated, using standard bench equipment.

As MacInnes and Taylor (2001) pointed out, commercially written software is seldom validated, because most products do not include source code for testing. Using a rig such as the one described, we are in the process of benchmarking commercial and noncommercial packages. The results of these tests, together with details of benchmarks, will be the subject of a separate paper.

In addition, it is our hope that individual researchers will submit their hardware and software to us for evaluation so that we can report on its timing accuracy and consistency, whether their paradigms be constructed by using experiment generators or custom-written by using a high-level programming language to perform a specific task.

\section{REFERENCES}

FinNey, S. A. (2001). Real-time data collection in Linux: A case study. Behavior Research Methods, Instruments, \& Computers, 33, 167 173.

MacInNes, W. J., \& TAYLOR, T. L. (2001). Millisecond timing on PCs and Macs. Behavior Research Methods, Instruments, \& Computers, 33, 174-178.

Myors, B. (1998). A simple graphical technique for assessing timer accuracy of computer systems. Behavior Research Methods, Instruments, \& Computers, 30, 454-456.

Myors, B. (1999). Timing accuracy of PC programs running under DOS and Windows. Behavior Research Methods, Instruments, \& Computers, 31, 322-328. 